\title{
Development of Fixed-base AHS Simulator for Human Factors Researches
}

\author{
Peom Park* Doo-Won Cha*, \\ ppark@madang.ajou.ac.kr·its@humanfactors.co.kr \\ Information and Industrial System Engineering Department, Ajou University \\ San5 Wonchong-Dong, Paldal-Gu, Suwon, Korea \\ Phone : +82-31-219-2426 - Fax : +82-31-219-1610
}

\begin{abstract}
This study describes the specifications and configurations of the fixed-base AHS simulator not only for the human factors researches, but also for the expansion of network-base multi-user AHS simulator for the investigation of individual drivers behaviors those effects on the traffic flow and AHS.
\end{abstract}

\section{Introduction}

AHS-fully automated driving-aims to automate the operations of the vehicles on the highway to eliminate the problems of traffic flow caused by operations of vehicles by individual drivers. It means that AHS increases highway safety by reducing driver error, which causes a large share of motor vehicle crashes, because the performance of driving depends on how the following environments of driving are perceived and translated into specific decisions; they are the weather condition, highway geometric design, and traffic flow conditions. As the results, not only the traffic flow can be made uniform, but also the operations can be controlled such that the flow is optimized in terms of capacity, energy use and environmental impacts. Further, the individual drivers are relieved of the driving tasks leading to the reduction of fatigue and judgment errors [6].

Human Factors considerations are crucial issues for AHS design because human driver should be involved in automated driving even if there is no need to control the steering and speed during automated driving mode. Because, for instance, drivers may be expected to instruct their vehicles and exit locations and destinations, take control in some emergency situations, and driving authority changes. Because of all roads will not be automated, drivers should control the vehicle manually before and after automated driving. Also, newly developed in-vehicle information systems essentially required to deliver the AHS, vehicle and traffic information, for example, head-up display, AHS flat panel display. Considering these kinds of changed driving maneuvering and driver-vehicle interfaces, the driver's capabilities and limitations must be considered to ensure the successful and safe implementation of the AHS in term of driver-centered. Human factors has performed important roles to investigations of AHS driver physical, demographical, psychological characteristics to provide the basis for determining system design configurations and features, and to develop the human factors design guidelines.

This study described the developed fixed-base AHS driving simulator and its applications for human factors researches.

\section{Development Objectives}

Generally, most of AHS-related human factors researches have been performed using large-scale driving simulator that is an expensive, elaborate, and inclusive tool that uses a combination of seat, steering wheel, and foot control to provide a realistic virtual driving environment, for example, Japan Automotive Research Institute Driving Simulator [11], Iowa university Driving Simulator [1][2], VTI driving simulator [4], Eva [8], and so on.

Developed AHS simulator is a fixed-base and low-cost simulator for the human factors evaluation that based on the shared space-at-grade road configurations and Korean road specification and the real road environments. Although, this simulator is a exclusive AHS simulator for suggested road configuration, this kind of simulator has widely used for the human factors and cognitive researches for ITS and transportation systems, for example human drivers route choice modeling, impacts analysis of newly development system, driver-vehicle interface development, workload and performance estimation, basic researches for large-scale driving simulator development, and so on. Because, this kind has the advantages of rather short development time, quick response from research needs, simple operations, higher extendibility, and quick change of experiment variables, easy replication by comparing than large-scale driving simulator, despite of driving environmental constraint [3].

This simulator included following objectives;

(1) To investigate the driver-centered inter-vehicle gap in a platoon

(2) To monitoring the drivers behavioral characteristics 
when using AHS

(3) To predict the imposed drivers workloads among road sections

(4) To analyze the impact of automated driving to the driver's performance

(5) To investigate the driving authority transfer control

(6) To evaluate the AHS usability and user's acceptance

(7) And others human factors researches and drivers monitoring

These kinds of works could help to suggest the basic human factors design guidelines for AHS driver-vehicle and road interface design, and this type of simulator could help for the development of network-based multi-driver simulator to investigate the driver's characteristics and their interactions to analyze the efficiency and traffic capacity of AHS. Various human factors evaluation tools were equipped that described in Chapter 4, and AHS speed can be controlled from $100 \mathrm{kph}$ to $200 \mathrm{kph}$ with $1 \mathrm{~m}$ to $20 \mathrm{~m}$ inter-vehicle gaps by manipulating the calibration module.

\section{Simulator Descriptions}

\subsection{Hardware and Software Specifications}

Simulator was constructed on a half-sized real vehicle cockpit module (Hyundai Verna automatic transmission model) with 16 d.o.f. real vehicle dynamics model. Virtual driving environment was injected using beam projector (Sanyo Pro xtraX multimedia projector) on the 110-inch size of screen.

This simulator has $45^{\circ} \sim 55^{\circ}$ field of view and distance from projector and screen was $3.6 \mathrm{~m}$ to present the real-size road environment and features with $800 \times 60032$ bits true color resolution through $40 \sim 60 \mathrm{~Hz}$ refresh rate. $3 \mathrm{D}$ vehicle sound was generated through four speakers around the vehicle using integrated control box. Host computer was Pentium III 700Mhz capability that controlled by keyboard and mouse connected with integrated control box. Flat panel display was implemented on the 7-inch LCD-based portable client computer display using IPX protocol for the synchronization between vehicle status and information on the display. Table 1 describes the hardware and software specifications, and Figure 1 shows the overall functional diagram of this simulator.

\subsection{Driver-Vehicle Interface}

Head-up display was implemented on the screen graphically, and the AHS flat panel display was implemented on the 7-inch LCD-based portable computer display. Head-up display presented the current vehicle position on the lanes, speed, driving mode (automated or manual driving), and rest time to destination, and driving status, for example the changing the lane, entering the automated lane and so on. And, flat panel display presented the rest distance to destination, inter-vehicle distance, destination, and driving mode on the rightside of the cockpit.
Eight variable message signs were implemented for the driver's rapid and exact information acquisition and reactions just before each gate and in front of $500 \mathrm{~m}$ of each gate with young female voice instructions. Figure 2 displays the screenshot of AL (Automated Lane) driving on this simulator. Five functions keys were arranged on the instrument panel area for the calibration of vehicle calibration (function key 1 4), and for the CCB (Cruise Control Button) for automated driving (function key 5 ). By pressing the CCB, subjects could start the automated driving on the transition lane. Figure 3 shows the driving scene and driver-vehicle interface of cockpit area with five function keys.

\subsection{Road Configurations and Driver's Maneuvering}

Figure 4 shows the road configuration that implemented in this simulator that based on the shared space at-grade concept suggested by PATH (Partnerships for Advanced Transit and Highways) [12]

Table 1. Summary of H/W and S/W Specification of AHS Simulator

\begin{tabular}{|l|l|}
\hline Hardware Specification & \multicolumn{1}{|c|}{ Software Specification } \\
\hline Computer System & Development Environment \\
- Main processor (CPU) : & Microsoft Visual Studio (Visual \\
Pentium III 700Mhz & C++6.0) \\
- Memory (RAM) : 256Mb & Graphics Library : Spectrum \\
- Graphic Card : Supports & Ver. 1.5 (OpenGL-based \\
Open GL, AGP 32Mb, Riva & Graphics Library) \\
TNT II G-Force (nVidia Co.) & - Client : Microsoft Foundation \\
- Sound Card : 3D Sound, PCI & Class \\
Signal IO Interface Card & Software Modules \\
(KITI IO Interface Card) & - Interface Processing Module \\
Integrated Control Box & - Calibration Module \\
- Sound Generation System & - Vehicle Dynamics Calculation \\
Four 3D Sound Speaker and & Module \\
Amplifier Input Device : & - Check/Inspection Module \\
Mouse and Keyboard & - Display Module \\
Display System : Beam & - Vehicle Generation Module \\
Projector and Screen (Sanyo & - Camera Calculation Module \\
Pro xtraX) & - Communication Module \\
Vehicle Cockpit & Menu Module \\
Hyundai Verna Automatic & \\
\hline
\end{tabular}

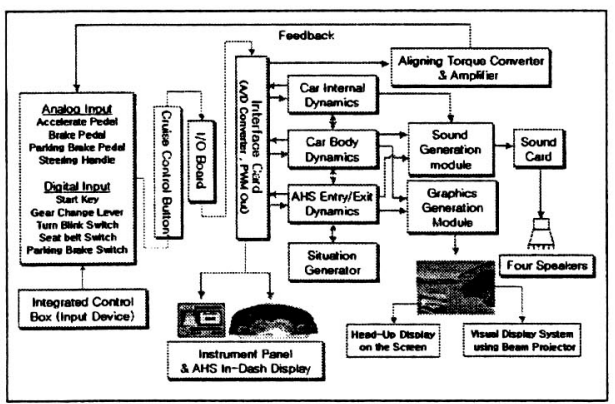

Figure 1. Overall Block Diagram of AHS Simulator 


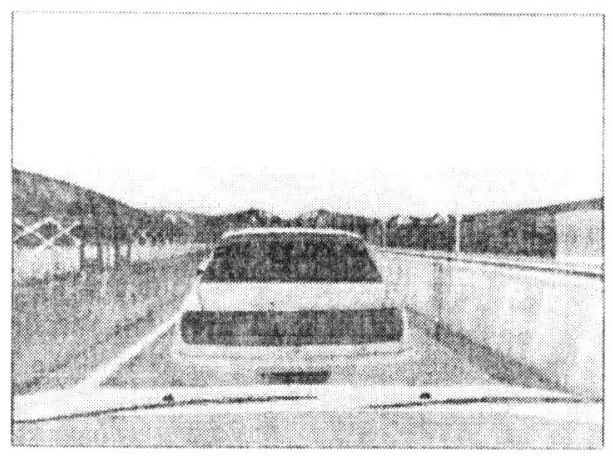

Fisure 2 implemented Kout fownoment

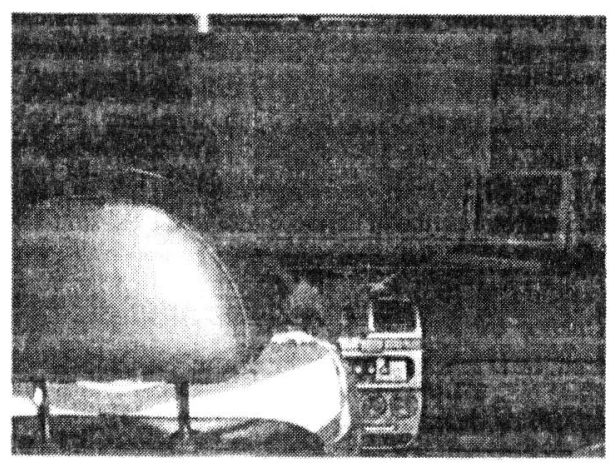

Figure3. Experiment Scene

This shared at-grade concept is a low-cost minimumchangeable configuration for the implementation of Korean road situation through a transportation expert's review. This concept also, satisfies the principles governing the configuration of the AHS, and the maneuvers of vehicles like below [5].

(1) Vehicles are organized into closely spaced platoons, which have an inherently low casualty rate

(2) There is a barrier (or divider) between the $\mathrm{AL}$ and $\mathrm{ML}$ (Manual Lane) on the rest of the right-of-way; vehicles must enter and exit through gates in the dividers

(3) Platoons do not join together (merge) at speed, either on the ALs or on the transition lane

To implement this concept on the virtual environment, graphic engineers modified recorded road scenes from Osan to Chonan. Barriers were used from Korean road facility standards [9], median (drawing number : II-15-2), AL-TL (Transition Lane) barrier and AL-TL barrier (drawing number : II-3-1) were used. And, road width was $3.5 \mathrm{~m}$ that is the minimum requirements from Korean regulations of road structure and facility [10]. In Figure 4, EML (Entry Maneuvering Length) is the area that the drivers press the CCB to start the automated driving and to check the vehicle state, $\mathrm{CCL}$ (Control Change Length) is for the driving authority transferring from vehicle to driver/driver to vehicle on the TL, and FML (Failure Maneuvering Length) gate is the exiting area in case of CCB press failures or vehicle malfunctions. During road sections, $\mathrm{CCL}$ length after exiting $\mathrm{AL}$ is long, because there is possibility that the driver fall into drowsiness and he or her should prepare the manual driving.

To complete a session of experiment, subject should drive the car by following orders; $\mathrm{ML} \rightarrow \mathrm{TL} \rightarrow \mathrm{AL} \rightarrow \mathrm{TL} \rightarrow$ $\mathrm{ML}$. When the subject enters the TL for AHS driving, vehicle control authority changed to the vehicle after pressing the $\mathrm{CCB}$ on the instrument panel. Then, the vehicle automatically enters the $A L$, increasing the vehicle velocity until the predetermined AHS speed at the end of platoon. In case of, the subject failed to push the CCB, they should driver the vehicle through the FML gate. At the end of automated driving on $\mathrm{AL}$, vehicle also automatically moves to the gate at the end of TL to change to control authority to the driver.

\section{Established Human Factors Evaluation Equipments}

Driver's behavior monitoring system, psychophysiological parameters measurement system, secondary task generation, and steering behavior data collection systems were equipped on the simulator basically. Figure 5 shows the experiment chamber and human factors apparatus settings.

Biopaq and TEL 100 system were estabiished to measure the driver's psychophysiological workloads, for example, galvanic skin response, electrooculogram, electroencephalograph, heart rate variability, electrocardiogram, and so on. A red lamp was set up on the dashboard and a push switch was placed on the $\mathrm{AC}$ register, and this lamp was connected with digital timer. When the red lamp turned on by experimenter, subject was required to push the switch as soon as possible on the ventilation to compare the drivers' reaction time among various driving conditions. Potential meter was installed on the steering wheel, then vehicle speed and steering angle data automatically logged in the host computer with the brake

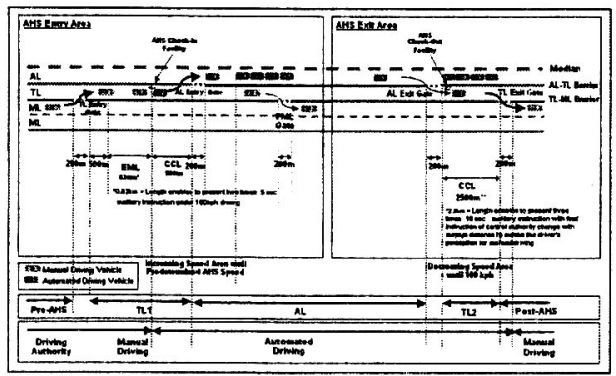

Figure4. Road Configuration 
and accelerator control behavior.

Four CCDs were equipped around the simulator. CCD1 recorded the driving scenes that including frame numbers, and CCD2 monitored driver's foot behavior to investigate the driver's authority transfer behavior. $\mathrm{CCD} 2$ was installed in front of subject to monitoring the driver's behavior. $\mathrm{CCD} 3$ recorded the reaction time of digital timer and processing time of Biopac system, and CCD4 was installed in front of the driver to monitor the driver's state and steering behaviors. All video data were recorded in a videotape using digital color quad switcher.

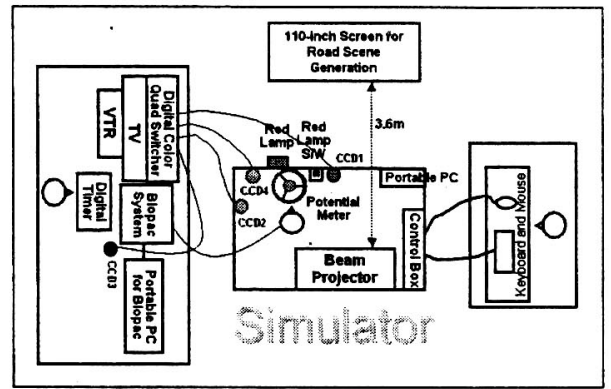

Figure5. Experiment Chamber and Apparatus Settings

\section{Conclusion : Applications and Extensions}

Korean Ministry of Construction and Transportation announced that they have established the plan to install the national-wide ITS until 2010, and also, they will develop and service the AHS until 2020 [7]. And, this study suggested the configurations and specifications of the AHS simulator as the basic tool of the human factors research and prototype for future extensions.

Firstly, using this simulator, it is possible to reveal the expected impact of automated driving on driver's workload and performance among road sections, and the usability evaluation of AHS would suggest the subjects preferences and opinions after their riding experiences of simulator. Also, these kinds of works could be performed by the various demographic subjects groups.

Secondly, the results of experiments using this simulator could assist the hardware and electronics engineers for the driver-vehicle interface design and evaluation by suggesting the human factors guidelines. Because, the R\&D policy and implementations of ITSrelated systems have been performed focused on the hardware developments in Korea.

For the further research, there strongly need the network-based multi-user driving simulator that is possible to combine with the various drivers cognitive and traffic models. This kind simulator could helpful to develop the optimal driver-vehicle interface design and workload evaluation, and to estimate the AHS effects on the traffic flow and capacity. Then, suggested simulator could be used as the prototype of for the future development by suggesting the simple Internet protocol-based extendibility and driver-vehicle interfaces.

\section{References}

[1] Bloomfield, J. R., Lee, Levitan, Angela R. Grant, Timothy L. Brown, Jon M. Hankey, Driving Performance After an Extended Period of Travel in an Automated Highway System, FHWA-RD-98- 051, 1998.

[2] Buck, R. J., Yenamendra, A., Ergonomics Issue on Entering the Automated Highway System, In: Noy, I. N (Ed.), Ergonomics and Safety of Intelligent Driver Interfaces, Lawrence Erlabaum Associates, Publishers, Mahwah, New Jersey, 1997.

[3] Cha D. W., Park P., "Development Human Factors Evaluation System for Car Navigation System", IEInterfaces, Vol. 12, No. 2, pp. 294-304, 1999.

[4] Gilling, S. P., Flynn, G. P., "Applications to Intelligent Transport Systems of the AC- ASSIST Project", Proceedings of 2nd World Congress of ITS

[5] Hitchcock, A., Configuration and Maneuvers in SafetyConsciously Designed AHS Configuration, California PATH Working Paper, UCB-ITS-PWP-95-2.

[6] Kikuchi, S., Tanaka, Y., AHS Planning : Evaluation of Reasoning Process , Proceedings of 4th World Congress on ITS, Berlin, Germany, 1997.

[7] Korean Ministry of Construction and Transportation Website, 2001.

[8] Mizutani, H., Hatanaka, K., Mashimo, H., SuetomiI, T., Yamada, Y., "Evaluation for AHS Architecture - Development of Traffic Simulator", Proceeding of 4th World Congress of ITS, Berlin, German, 1997

[9] Regulations of Road Structure and Facility, Korean Ministry of Construction and Transportation, 1999.

[10] Road Attendant Facilities Standards, Korean Ministry of Construction and Transportation, 1998.

[11] Suetomi, T., Hashimoto, M., Iwao, M., Iwama, T., "Driver Evasive Maneuver in Advanced CruiseAssist Highway System (AHS)", Proceeding of 7th World Congress of ITS, Toronto, Canada, 2000.

[12] Yim, Y., Miller, M. A., Hellaman, P., Sharafsaleh, M., Integration of Automated Highway Systems into Existing California Freeways, Petros A Ioannou (Eds.), Auto- mated Highway Systems, pp.29-48, Plenum Press, New York, 1997. 